\title{
SIMULAÇÕES DE PROCESSOS NUCLEARES EM EXPLOSÕES SOLARES UTILIZANDO O PACOTE MONTE CARLO FLUKA
}

\author{
Daneele S. Tusnski \\ Sergio Szpigel \\ Carlos Guillermo Giménez de Castro \\ Universidade Presbiteriana Mackenzie (UPM)
}

\begin{abstract}
Resumo
A emissão de raios gama em explosões solares é produzida por interaçóes de elétrons e íons acelerados a altas energias com núcleos da atmosfera solar ambiente. A análise dos espectros de emissão de raios gama observados em explosóes solares fornece diagnósticos importantes tanto dos mecanismos de aceleração das partículas quanto da estrutura e da evoluçáo do plasma ambiente. Neste trabalho, apresentamos e discutimos os resultados de simulaçóes de processos nucleares de alta energia em explosóes solares utilizando o pacote Monte Carlo FLUKA. Considerando modelos simples para a atmosfera solar ambiente e feixes de prótons primários acelerados com diferentes distribuições energéticas e angulares, obtivemos espectros de emissão de raios gama semelhantes aos observados em explosóes solares.
\end{abstract}

Palavras-chave: Explosão solar. Processos nucleares. FLUKA. 


\section{INTRODUÇÃO}

As explosóes solares são eventos transientes e intensos que ocorrem nas chamadas regiôes ativas da atmosfera solar, constituídas por um plasma com intensos campos magnéticos estruturados em configuraçôes complexas de arcos magnéticos e que resultam em um abrilhantamento repentino observado no disco solar ou no limbo (TANDBERG-HANSSEN; EMSLIE, 2009). Esses eventos envolvem a liberação de energias da ordem de $10^{27}$ a $10^{32}$ ergs em poucas dezenas de segundos até dezenas de minutos. Segundo o modelo padrão geralmente adotado para descrever as explosóes solares (DULK; MARSH, 1982; BASTIAN; BENZ; GARY, 1998), a energia armazenada nos campos magnéticos é liberada através do processo denominado reconexão magnética, que aquece o plasma magneto-ativo e acelera elétrons e íons (prótons, partículas alfa e íons mais pesados) até altas energias. Esse processo resulta na emissão de radiação praticamente em todas as faixas do espectro eletromagnético, de ondas de rádio até raios gama. $\mathrm{O}$ plasma magneto-ativo aquecido produz emissão de radiação ultravioleta e emissão de raios $\mathrm{X}$ moles por bremsstrahlung de elétrons térmicos (emissão free-free). Os elétrons acelerados (não térmicos) produzem emissão de radiação sincrotrônica na faixa de rádio e micro-ondas e emissão contínua de raios $\mathrm{X}$ duros e raios gama por bremsstrahlung (DULK; MARSH, 1982). Os íons acelerados produzem emissão na faixa de raios gama por meio de processos nucleares.

A primeira previsão de que reaçóes nucleares que ocorrem em explosóes solares poderiam produzir raios gama detectáveis da Terra foi publicada por Morrison (1958). Posteriormente, uma teoria detalhada foi estabelecida por Lingenfelter e Ramaty (1967) para o cálculo da emissáo contínua e de linhas de raios gama esperadas, bem como de nêutrons de alta energia. Desde a publicação desses trabalhos pioneiros, a teoria para o tratamento da emissão de raios gama em explosôes solares tem sido significativamente refinada (RAMATY; MURPHY, 1987; VILMER; MACKINNON; HURFORD, 2011).

As quantidades mensuráveis associadas às emissôes de alta energia em explosóes solares fornecem informações sobre os elétrons e íons acelerados que as produzem, descritas pelos parâmetros de aceleração (RAMATY, 1995; MURPHY et al., 2007). Por exemplo, o ajuste do espectro de emissão contínua de raios $\mathrm{X}$ duros produzidos por bremsstrahlung de elétrons permite obter o número de elétrons primários acelerados e as características de sua distribuição espectral de energia. Da mesma forma, a medida das intensidades absolutas e relativas das linhas de raios gama produzidas por desexcitação nuclear permite obter o número de prótons e partículas alfa acelerados e 
as características de suas respectivas distribuiçôes espectrais de energia, bem como a abundância relativa de íons mais pesados acelerados. No entanto, as partículas aceleradas são transportadas ao longo dos arcos magnéticos desde o local de aceleração até a região de interação. Assim, as quantidades mensuráveis associadas às emissóes de alta energia também dependem fortemente das condiçôes físicas da atmosfera solar ambiente e da estrutura dos arcos magnéticos, descritas por parâmetros físicos como a localização da explosão, a composição e os perfis de densidade e temperatura da atmosfera solar durante a explosão, o comprimento do arco magnético e o grau de convergência do campo magnético nos pés do arco. Portanto, a análise das emissóes de alta energia em explosões solares fornece diagnósticos importantes tanto dos mecanismos de aceleração das partículas quanto da estrutura e evolução do plasma ambiente (MURPHY et al., 2007). É relevante observar que a emissão de raios gama fornece informaçóes sobre os elétrons e os íons acelerados, uma vez que o espectro na faixa de raios gama apresenta a contribuição do contínuo produzido por bremsstrahlung de elétrons acelerados e a das linhas de desexcitaçáo nuclear, enquanto o espectro na faixa de raios $\mathrm{X}$ duros apresenta somente a contribuição do contínuo devido aos elétrons, sem assinaturas significativas da presença dos íons (ASCHWANDEN, 2005).

Neste trabalho, apresentamos e discutimos os resultados de simulaçóes de processos nucleares de alta energia que ocorrem em explosóes solares utilizando o FLUKA (FERRARI; SALA; FASSÒ; RANFT, 2011), um pacote integrado de rotinas de uso geral desenvolvido para o cálculo Monte Carlo do transporte e das interaçóes de partículas na matéria. O objetivo é validar o pacote FLUKA como ferramenta efetiva para a modelagem de processos nucleares no contexto de explosôes solares. Para tanto, realizamos simulaçóes do espectro de emissão de raios gama considerando modelos simples para a atmosfera solar ambiente e feixes de prótons acelerados com diferentes distribuições energéticas e angulares.

$\mathrm{Na}$ seção 2 deste artigo descrevemos as principais características do espectro de emissão de raios gama em explosóes solares e os processos nucleares envolvidos em sua produção. Na seção 3, apresentamos as principais características do pacote FLUKA e descrevemos os modelos utilizados nas simulaçóes. Na seção 4, apresentamos e discutimos os resultados obtidos. $\mathrm{Na}$ seção 5, por fim, apresentamos as conclusóes e consideraçôes finais. 


\section{ESPECTRO DE EMISSÃO DE RAIOS GAMA EM EXPLOSÓES SOLARES}

\subsection{Características gerais}

A Figura 1 mostra um espectro de emissão típico de uma explosão solar intensa. $\mathrm{Na}$ faixa de raios $\mathrm{X}$ moles ( $1 \mathrm{a} 10 \mathrm{keV})$, o espectro é dominado pela emissão contínua de radiação produzida por bremsstrahlung de elétrons térmicos, apresentando também linhas de emissão produzidas por transiçôes atômicas. $\mathrm{Na}$ faixa de raios $\mathrm{X}$ duros $(\sim 10 \mathrm{keV}$ a $1 \mathrm{MeV})$, o espectro é dominado pela emissão contínua de radiação produzida por bremsstrahlung de elétrons não térmicos relativísticos. Nessa faixa, nota-se também a presença da linha de aniquilação elétron-pósitron em $511 \mathrm{keV}$. Na faixa de raios gama ( $1 \mathrm{MeV}$ a $100 \mathrm{GeV})$, o espectro apresenta componentes devido à emissão contínua de radiação produzida por bremsstrahlung de elétrons não térmicos ultrarrelativísticos, à emissão de linhas de desexcitação nuclear (no intervalo de energia de $\sim 0,5$ a $10 \mathrm{MeV}$ ) e à emissão contínua de radiação produzida pelo decaimento de píons neutros (para energias maiores que $\sim 10 \mathrm{MeV}$ ). Na faixa de raios gama, nota-se também a presença da linha de captura de nêutrons em 2,223 MeV.

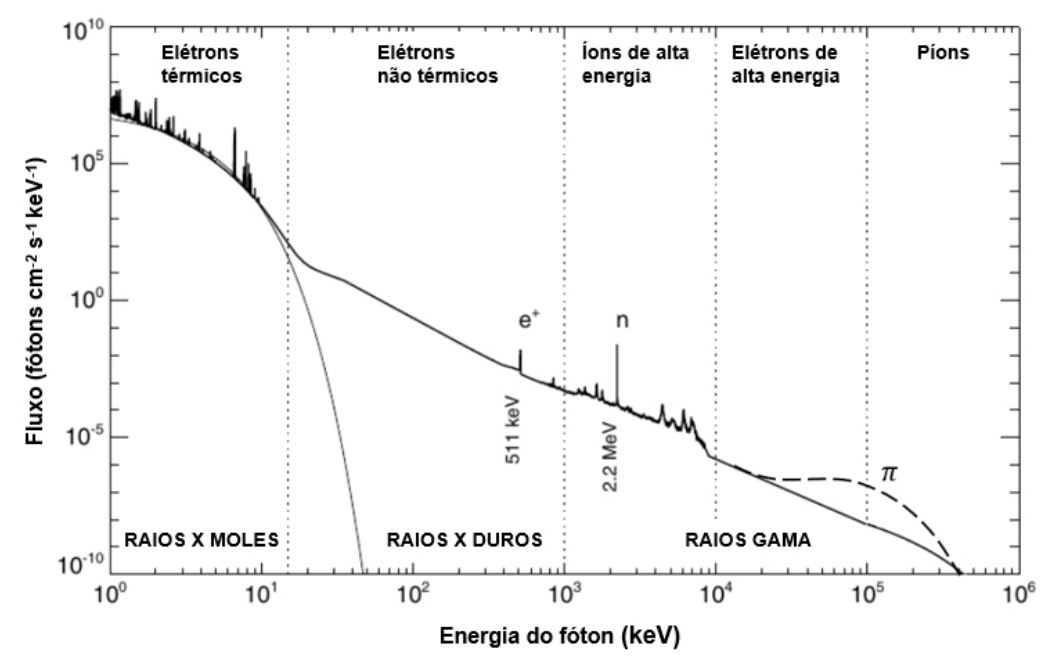

Figura 1 Espectro de emissão típico de uma explosão solar.

Fonte: Adaptado de Aschwanden (2005). 


\subsection{Processos nucleares em explosóes solares}

A emissão de raios gama em explosões solares é produzida por processos nucleares envolvendo colisóes de íons primários (prótons, partículas alfa e núcleos mais pesados) acelerados a altas energias $(\sim \mathrm{MeV}$ até $\mathrm{GeV})$ com núcleos da atmosfera solar ambiente. Revisóes detalhadas sobre os processos nucleares em explosóes solares podem ser encontradas em Ramaty e Murphy (1987) e Vilmer, MacKinnon e Hurford (2011).

Íons acelerados com energias na faixa de $\sim 1$ a $100 \mathrm{MeV} /$ nucleon podem produzir núcleos excitados cuja desexcitação produz a emissão de linhas estreitas e largas de raios gama na faixa de $\sim 0,5$ a $10 \mathrm{MeV}$ (RAMATY; KOZLOVSKY; LINGENFELTER, 1979). As linhas estreitas são produzidas por colisôes de prótons e partículas alfa acelerados com núcleos da atmosfera solar ambiente. As linhas largas são produzidas por reaçóes inversas em que núcleos mais pesados acelerados, como carbono e oxigênio, colidem com núcleos de hidrogênio e hélio da atmosfera solar ambiente. Íons acelerados com energias na faixa de $\sim 1$ a $100 \mathrm{MeV} /$ nucleon também podem produzir núcleos radioativos beta-emissores, cujo decaimento produz elétrons e pósitron secundários com energias típicas de $\sim 1 \mathrm{MeV}$ (KOZLOVSKY; LINGENFELTER; RAMATY, 1987).

Íons acelerados com energias maiores que $\sim 200-300 \mathrm{MeV}$ podem produzir píons secundários que contribuem para a emissão de radiação gama contínua mediante processos de decaimento (MURPHY; DERMER; RAMATY, 1987). Píons neutros decaem diretamente em dois fótons de energia 67,5 MeV ou, mais raramente (razão de ramificação $\sim 1,2 \%$ ), em um fóton e um par elétron-pósitron por meio do chamado decaimento Dalitz (OLIVE, 2014). Píons carregados (positivos e negativos) decaem em múons, cujo decaimento subsequente produz pósitrons e elétrons secundários que emitem radiação gama contínua por bremsstrahlung. Pósitrons secundários também produzem radiação gama contínua por aniquilação em voo e a linha em $511 \mathrm{keV}$, por sua vez, por meio da aniquilação em repouso (MURPHY et al., 2005).

As interaçóes dos íons acelerados com núcleos da atmosfera solar ambiente também podem produzir nêutrons secundários, que se propagam para fora do Sol ou precipitam na fotosfera. Uma parcela dos nêutrons que precipitam na fotosfera é capturada por átomos de hidrogênio (após termalizaçáo) produzindo deutério, processo que resulta na emissão da linha em 2,223 MeV (HUA et al., 2002). 


\section{DESCRIÇÃO DOS MÉTODOS COMPUTACIONAIS E MODELOS}

\subsection{Pacote FLUKA}

O FLUKA (FERRARI; SALA; FASSÒ; RANFT, 2011; BATTISTONI et al., 2015) é um pacote integrado de rotinas de uso geral para o cálculo Monte Carlo do transporte e das interaçóes de partículas na matéria. O pacote tem sido utilizado no desenvolvimento de aplicaçôes em diversas áreas, como física experimental de altas energias, dosimetria, design de telescópios e detectores, raios cósmicos, calorimetria e física médica.

O FLUKA pode simular com alta precisão o transporte e as interaçôes de aproximadamente 60 tipos diferentes de partículas, incluindo elétrons e múons com energias de $1 \mathrm{keV}$ até $1000 \mathrm{TeV}$, fótons com energias de $100 \mathrm{eV}$ até $10000 \mathrm{TeV}$, hádrons com energias de $1 \mathrm{keV}$ até $10000 \mathrm{TeV}$ e todas as antipartículas correspondentes, bem como neutrinos, nêutrons de baixa energia $(<20 \mathrm{MeV})$ e íons pesados com energias até $10000 \mathrm{TeV} /$ nucleon. As interaçóes são implementadas utilizando-se modelos físicos robustos e modernos, continuamente verificados e otimizados por meio da comparação com dados experimentais no nível de interaçóes individuais.

Os modelos utilizados no FLUKA para o tratamento de partículas eletromagnéticas (elétrons, pósitrons e fótons) e múons cobrem um amplo conjunto de processos, incluindo bremsstrahlung, espalhamento Compton e Rayleigh, ionização, produção de pares, aniquilação de pósitrons, efeito fotoelétrico, bem como interaçóes fotonucleares (FERRARI; SALA; GUARALDI; PADOANI, 1992). O transporte e as perdas de energia por ionização são implementados pelo meio de uma abordagem comum a todas as partículas carregadas, embasada na teoria de Bethe-Bloch (BETHE; HEITLER, 1934; BETHE, 1953).

O tratamento das interaçóes hadrônicas no FLUKA é baseado em uma abordagem microscópica em que são utilizados vários modelos ajustados aos diferentes intervalos de energia envolvidos (FERRARI; SALA, 1998; BATTISTONI et al., 2015). Em energias abaixo de $5 \mathrm{GeV}$, interaçôes inelásticas hádron-hádron são implementadas por meio de um modelo embasado na produção e no decaimento ressonante de partículas, enquanto interaçóes elásticas e de troca de carga são implementadas por meio da análise dos deslocamentos de fase e da aproximação eikonal. Em energias no intervalo de 5 a $20 \mathrm{TeV}$, as interaçóes inelásticas hádron-hádron são implementadas pelo Dual Parton Model (DPM) acoplado a um esquema de hadronização (CAPELLA et al., 1994). 
As interações inelásticas hádron-núcleo no intervalo de energia do limiar de reação até $20 \mathrm{TeV}$ são implementadas por meio do modelo do FLUKA denominado PEANUT (PreEquilibrium Approach to NUclear Thermalization) (FERRARI; SALA, 1994; FASSÒ et al., 1994; FERRARI; SALA, 1998; BATTISTONI et al., 2006). O PEANUT descreve as interações entre hádrons e núcleos como uma sequência dos seguintes passos:

1. cascata de espalhamentos múltiplos (modelo de Glauber - Gribov);

2. cascata intranuclear generalizada (GINC - Generalized IntraNuclear Cascade);

3. estágio pré-equilíbrio (emissão baseada em éxcitons);

4. estágio de equilíbrio (incluindo os processos de evaporação, fissão, break-up de Fermi e emissão de raios gama por desexcitação nuclear).

A extensão para o tratamento de interações núcleo-núcleo é implementada por meio de três geradores de eventos externos conectados ao FLUKA, baseados, respectivamente, nos seguintes modelos: Boltzmann Master Equation (BME) (CERUTTI, et al., 2006) em energias abaixo de $0,125 \mathrm{GeV} /$ nucleon; Relativistic Quantum Molecular Dynamics (RQMD - 2.4) (SORGE; STÖKER; GREINER, 1989; ANDERSEN et al., 2004) em energias de 0,125 a $5 \mathrm{GeV} /$ nucleon; Dual Parton Model and JETs (DPMJET-III) (ROESLER; ENGEL; RANFT, 2001) em energias acima de $5 \mathrm{GeV} /$ nucleon.

$\mathrm{O}$ transporte e as interaçôes de nêutrons de baixa energia $(<20 \mathrm{MeV})$, responsáveis pela emissão da linha de captura de nêutrons em 2,223 MeV, é implementado no FLUKA com um algoritmo baseado no método de multigrupos (FERRARI; SALA; FASSÒ; RANFT, 2011). As matrizes de espalhamento para os canais de reação são calculadas a partir de dados de seçóes de choque de nêutrons fornecidos por uma biblioteca dedicada, na qual o intervalo de energia de interesse é dividido em 260 grupos de nêutrons e 42 grupos gama.

\subsection{Modelos para simulaçáo dos processos nucleares}

Para simular os processos nucleares de alta energia em explosóes solares com o FLUKA, consideramos um modelo em que um feixe de prótons primários acelerados é injetado em um alvo com características similares às da atmosfera solar ambiente. Adotamos uma geometria planar simples, uma vez que as dimensóes da região de interação e emissão em uma explosão solar são sempre muito menores que o raio solar. 


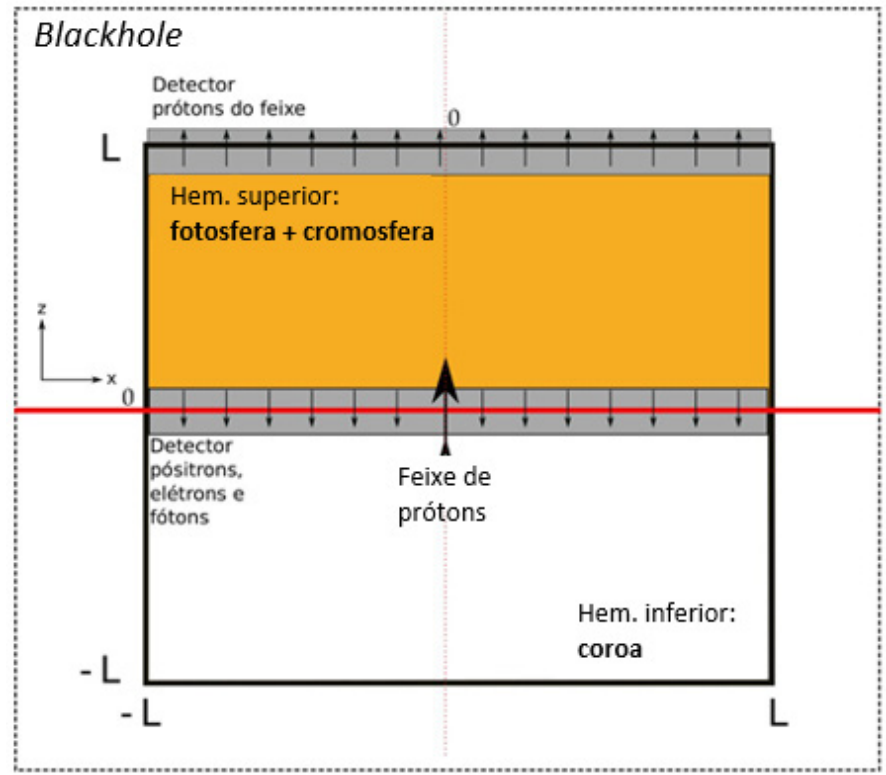

Figura 2 Representação em duas dimensões da geometria utilizada nas simulações.

Fonte: Elaborada pelos autores.

A Figura 2 mostra uma representação em duas dimensóes da geometria utilizada nas simulaçóes, na qual a região de interação e emissão da atmosfera solar ambiente é aproximada por um cubo de aresta $2 \mathrm{~L}$ com centro na origem de um sistema de coordenadas cartesianas $(\mathrm{Ox}, \mathrm{Oy}, \mathrm{Oz})$ e faces orientadas perpendicularmente aos eixos coordenados. O cubo é dividido em dois hemisférios por um plano (xy) situado em $\mathrm{z}=0$. $\mathrm{O}$ hemisfério superior $(\mathrm{z}>0)$ corresponde à região cromosférica/fotosférica e o hemisfério inferior $(\mathrm{z}<0)$ à região coronal. $\mathrm{O}$ cubo é inserido no interior de um cubo maior, representado pela linha tracejada, definindo a região em que as partículas não são mais rastreadas, denominada no FLUKA como blackhole.

Conforme indica a Figura 2, o feixe de prótons primários é injetado em direção à regiáo cromosférica/fotosférica $(\mathrm{z}>0)$ a partir de um ponto $\left(\mathrm{x}=0, \mathrm{y}=0, \mathrm{z}=-\mathrm{z}_{0}\right)$ situado na regiáo coronal $(\mathrm{z}<0)$ a uma distância $\mathrm{z}_{0}$ muito próxima do plano $(\mathrm{xy}) \mathrm{em}$ $\mathrm{z}=0$. Nas simulaçóes realizadas neste trabalho, consideramos feixes contendo $10^{7}-10^{8}$ prótons monoenergéticos ou seguindo uma distribuição de energia do tipo lei de potência em um dado intervalo de energia $E_{\min }<E<E_{\max }$, dada por:

$$
\frac{d n_{p}(E)}{d E}=A E^{-\delta}
$$


onde $\delta$ é o índice espectral e $A$ é uma constante de normalização, tal que:

$$
\int_{E_{\min }}^{E_{\max }} \frac{d n_{p}(E)}{d E} d E=1
$$

Para gerar as distribuiçôes de energia do tipo lei de potência, utilizamos uma versão modificada da sub-rotina SOURCE.f disponível no FLUKA, desenvolvida por MacKinnon, Szpigel, de Castro e Tuneu (2016). A sub-rotina também permite gerar feixes de partículas primárias com diferentes tipos de distribuição angular, incluindo as opçóes de feixe colimado (unidirecional, paralelo ao eixo $\mathrm{Oz}$ ) e de feixe com distribuição semi-isotrópica no hemisfério superior consideradas no presente trabalho.

Dependendo da natureza do plasma ambiente na região de interação e emissão, dois cenários podem ser considerados para simular o transporte de partículas aceleradas:

- Alvo espesso, no qual as partículas são injetadas em um plasma denso, perdendo energia por colisôes elásticas ou inelásticas até atingirem o repouso;

- Alvo fino, no qual as partículas são injetadas em um plasma tênue, de modo que não ocorrem perdas significativas de energia.

Consideremos uma partícula injetada em um alvo com perfil de densidade $\rho(z)$ na direção do eixo Oz. A profundidade de coluna (column-depth) do alvo é dada por:

$$
h_{c o l}=\int_{0}^{z_{\max }} \rho(z) d z,
$$

onde $z_{\max }$ denota a dimensão do alvo na direção do eixo $\mathrm{Oz}$.

A taxa média de perda de energia da partícula por unidade de distância percorrida no alvo ao longo do eixo $\mathrm{Oz}$ (geralmente expressa em $\mathrm{MeV} . \mathrm{cm}^{2} / \mathrm{g}$ ) é denominada stopping-power, sendo dada por (GETACHEW, 2007):

$$
S=-\frac{1}{\rho(z)} \frac{d E}{d z} .
$$

A distância média percorrida pela partícula ao longo do eixo $\mathrm{Oz}$ até atingir o repouso (geralmente expressa $\mathrm{em} \mathrm{g} / \mathrm{cm}^{2}$ ) é denominada stopping-depth ou range. $\mathrm{Na}$ aproximação de perda contínua de energia (Continuous Slowing-Down Approximation - CSDA), o range $R\left(E_{0}\right)$ de uma partícula com energia inicial $E_{0}$ é dado por: 


$$
R\left(E_{0}\right)=\int_{0}^{E_{0}} \frac{d E}{S} .
$$

Assim, para simular o transporte de partículas aceleradas em um cenário de alvo espesso, deve-se satisfazer a condição $h_{c o l} \geq R\left(E_{0}^{\max }\right)$, onde $R\left(E_{0}^{\max }\right)$ corresponde ao CSDA - range das partículas injetadas de maior energia, de modo que todas as partículas atravessando o alvo atingem o repouso. A condição para se obter um cenário de alvo fino, no entanto, é dada por $h_{c o l} \ll R\left(E_{0}^{\max }\right)$, tal que as partículas atravessam o alvo praticamente sem perder energia, i. e. $d E / E \ll 1$.

Consideramos dois modelos para a atmosfera solar ambiente que preenche o cubo. No Modelo I, assumimos que o hemisfério superior (regiāo cromosférica/fotosférica) é preenchido por uma atmosfera de densidade uniforme composta por $\mathrm{H},{ }^{4} \mathrm{He}, \mathrm{C}, \mathrm{N}$ e $\mathrm{O}$ com abundâncias relativas ao $\mathrm{H}$ dadas pelo modelo de Asplund et al. (2009), conforme indicadas na Tabela 1. No hemisfério inferior (regiáo coronal), assumimos por simplicidade que a densidade é nula (vácuo), uma vez que as densidades coronais são extremamente baixas $\left(<10^{-10} \mathrm{~g} / \mathrm{cm}^{3}\right)$.

\section{TABELA 1}

Composição da atmosfera solar ambiente utilizada nos Modelos I e II, com abundâncias relativas ao $\mathrm{H}$ dadas pelo modelo de Asplund et al. (2009).

\begin{tabular}{ccc} 
Elemento & Abundância relativa \\
H & 1,0 \\
${ }^{4} \mathrm{He}$ & 0,085 \\
C & 0,000245 \\
N & 0,000603 \\
\hline & 0,000457 \\
\hline
\end{tabular}

Fonte: Elaborada pelos autores.

Para simular um alvo espesso, utilizamos um cubo de aresta $2 \mathrm{~L}=2,0 \times 10^{7} \mathrm{~cm}$ com o hemisfério superior preenchido por uma atmosfera de densidade $\rho=3,19 \times$ $10^{-5} \mathrm{~g} / \mathrm{cm}^{3}$, tal que a profundidade de coluna obtida é $h_{c o l}=\rho L=319 \mathrm{~g} / \mathrm{cm}^{2}$. Dessa forma, a condição de alvo espesso é satisfeita, uma vez que o CSDA - range para prótons em um alvo de hidrogênio é $R(1 \mathrm{GeV})=158,7 \mathrm{~g} / \mathrm{cm}^{2}$ (BERGER et al., 2005). Para simular um alvo fino, utilizamos um cubo de aresta $2 \mathrm{~L}=2,0 \times 10^{5} \mathrm{~cm}$ com o hemisfério superior preenchido por uma atmosfera de densidade $\rho=3,19 \times 10^{-6} \mathrm{~g} / \mathrm{cm}^{3}$, tal que a profundidade de coluna obtida é $h_{c o l}=\rho L=0,319 \mathrm{~g} / \mathrm{cm}^{2}(\ll R(1 \mathrm{GeV}))$. 
No Modelo II, assumimos que ambos os hemisférios são preenchidos por uma atmosfera com composiçáo dada pela Tabela 1 e consideramos um perfil de densidade na direção do eixo $\mathrm{Oz}$ dado pela aproximação analítica de Kotoku et al. (2007) para o modelo Harvard-Smithsonian da atmosfera solar,

$$
\rho(z)=3,19 \times 10^{-7} \exp \left(\frac{z}{h}\right)\left(\mathrm{g} / \mathrm{cm}^{3}\right)
$$

onde $z$ corresponde à coordenada medida $(\mathrm{em} \mathrm{km}$ ) a partir do plano $(\mathrm{xy}) \mathrm{em} \mathrm{z}=0 \mathrm{e}$ h denota uma escala de altura cujo valor é $110 \mathrm{~km}$ para $z<0$ (região coronal) e $400 \mathrm{~km}$ para $z>0$ (região cromosférica/fotosférica).

A Figura 3 mostra o perfil de densidade considerado no Modelo II. Na região do hemisfério inferior entre os planos (xy) em z $=-2,0 \times 10^{9} \mathrm{~cm} \mathrm{e} \mathrm{z}=-1,0 \times 10^{9} \mathrm{~cm}$ a densidade é nula (vácuo). A região entre os planos (xy) em $\mathrm{z}=-1,0 \times 10^{9} \mathrm{~cm}$ e z $=0$ é dividida em cinco camadas, cada uma com densidade dada pela Eq. (6) e espessura determinada de modo a obter uma profundidade de coluna de $0,7 \mathrm{~g} / \mathrm{cm}^{2}$. O hemisfério superior, delimitado pelos planos (xy) em z $=0$ e $\mathrm{z}=-1,005 \times 10^{8} \mathrm{~cm}$, é dividido em 10 camadas com profundidade de coluna de $3,5 \mathrm{~g} / \mathrm{cm}^{2}$ e 4 camadas com profundidade de coluna de $30 \mathrm{~g} / \mathrm{cm}^{2}$. Somando-se as profundidades de coluna correspondentes aos dois hemisférios, obtemos uma profundidade de coluna total $h_{c o l}=158,5 \mathrm{~g} / \mathrm{cm}^{2}$, de modo que para prótons primários com energias de até $\sim 1 \mathrm{GeV}$ temos um cenário de alvo espesso.
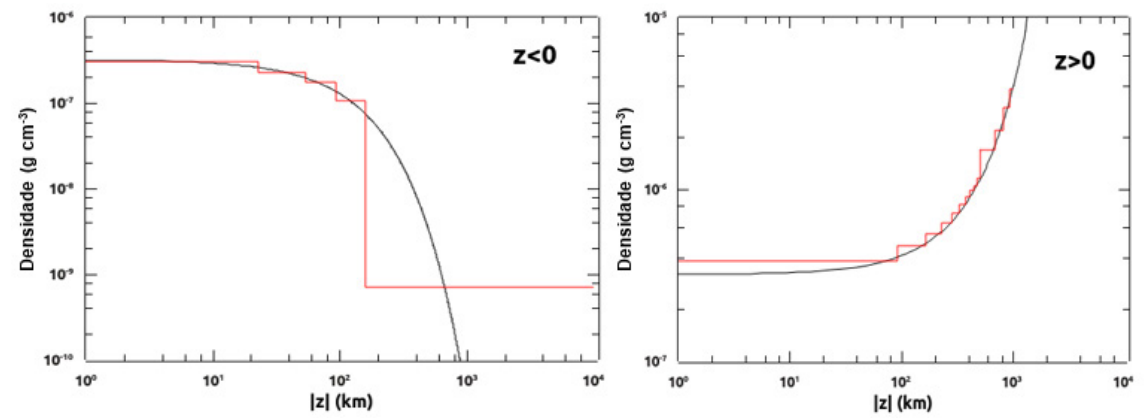

Figura 3 Perfil de densidade na direção do eixo Oz considerado para a atmosfera do Modelo II (vermelho), comparado à aproximação analítica dada pela Eq. (6) (preto). Painel esquerdo: região coronal. Painel direito: região cromosférica/fotosférica.

O FLUKA fornece diversas ferramentas de estimativa denominadas "detectores" com características e funcionalidades diferentes. Nas simulações de processos nucleares 
em explosôes solares realizadas considerando o modelo descrito acima, utilizamos detectores específicos (denominados USRBDX) para determinar o espectro de energia dos fótons que escapam da região cromosférica/fotosférica para a região coronal através do plano $(\mathrm{xy})$ em $\mathrm{z}=0$. Esses detectores permitem estimar a distribuição diferencial dupla do fluxo médio de partículas através de uma superfície por intervalo de energia e de angulo sólido, $\mathrm{d}^{2} \phi / \mathrm{dEd} \Omega$, em unidades de $\mathrm{GeV}^{-1} \cdot \mathrm{cm}^{-2} \cdot \mathrm{sr}^{-1}$ por partícula primária injetada. Assim, integrando o fluxo médio de fótons através do plano (xy) em $\mathrm{z}=0$ em ângulo sólido e assumindo detectores com área efetiva de $1 \mathrm{~cm}^{2}$, obtemos o espectro de energia correspondente, em unidades de $\mathrm{GeV}^{1}$ por partícula primária injetada.

\section{RESULTADOS}

No que se segue, apresentamos os resultados de simulaçóes realizadas com o pacote FLUKA utilizando os Modelos I e II descritos na seção 3.2. Comparamos os resultados obtidos para um alvo espesso e um alvo fino, para um feixe de prótons primários colimado e um feixe com distribuição angular semi-isotrópica no hemisfério superior e para um feixe de prótons primários monoenergéticos e um feixe com distribuição de energia do tipo lei de potência. Também analisamos o comportamento do espectro de energia dos fótons que escapam da regiáo cromosférica/fotosférica para a região coronal através do plano $(\mathrm{xy}) \mathrm{em} \mathrm{z}=0$ em relaçáo aos processos de bremsstrahlung de elétrons e pósitrons secundários, espalhamento Compton e decaimento de píons. Finalmente, comparamos os espectros de fótons obtidos em simulaçóes realizadas com os Modelos I e II.

A Figura 4 mostra as distribuiçóes espaciais de prótons primários na região de interação e emissão, projetadas no plano $(\mathrm{xz})$, obtidas para um alvo espesso e um alvo fino. Em ambos os casos, consideramos o Modelo I para a atmosfera solar ambiente e um feixe colimado de prótons primários monoenergéticos de $1 \mathrm{GeV}$. Conforme esperado, observamos que, no caso do alvo espesso, os prótons primários perdem totalmente sua energia ao atravessarem a região de interaçáo e emissão, e no caso do alvo fino praticamente todos os prótons primários escapam através da face superior deste. Em ambos os casos, podemos notar uma maior concentração de prótons primários ao longo do eixo $\mathrm{Oz}$, que corresponde à direção do feixe injetado. 

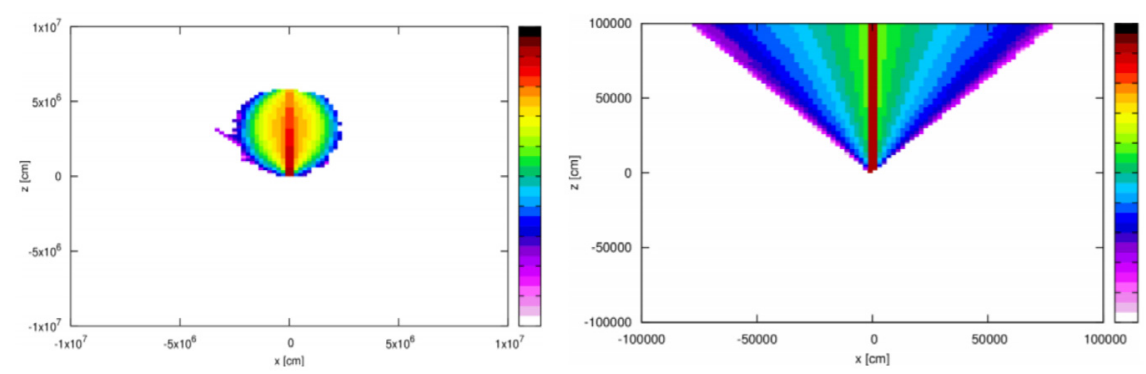

Figura 4 Distribuições espaciais de prótons primários, projetadas no plano (xz), obtidas para um alvo espesso (esquerda) e um alvo fino (direita).

A Figura 5 mostra os espectros de fótons obtidos para um alvo espesso e um alvo fino. Observamos que o fluxo de fótons obtido para o alvo espesso é mais intenso do que o obtido para o alvo fino. No caso do alvo espesso, notamos também a presença da linha de aniquilação em $511 \mathrm{keV}$, da linha de captura de nêutrons em 2,223 MeV e da componente de emissão contínua resultante do decaimento de píons na região de energias mais altas. No caso do alvo fino, observamos apenas a contribuição proveniente do decaimento de píons neutros com uma cauda na regiáo de energias mais baixas devida aos efeitos de transporte. A ausência das contribuiçóes provenientes do decaimento de píons carregados no caso do alvo fino deve-se ao fato de que a maioria dos múons produzidos pelo decaimento dos píons escapa da região cromosférica/fotosférica antes de decair em elétrons e pósitrons que produzem emissão contínua por bremsstrablung.

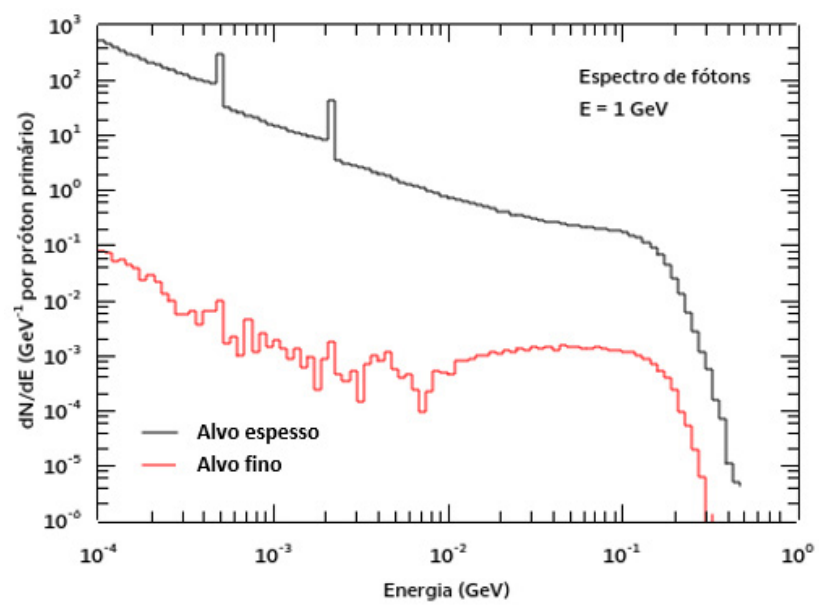

Figura 5 Espectros de fótons obtidos para um alvo espesso e um alvo fino. 
A Figura 6 mostra as distribuiçóes espaciais de prótons primários na região de interação e emissão, projetadas no plano (xz), obtidas para um feixe colimado e um feixe com distribuição angular semi-isotrópica no hemisfério superior. Em ambos os casos consideramos o Modelo I para a atmosfera solar ambiente, um feixe de prótons primários monoenergéticos de $1 \mathrm{GeV}$ e um cenário de alvo espesso. Observamos que, no caso do feixe com distribuição semi-isotrópica, um número significativo de prótons primários é espalhado de volta à região coronal, devido a colisôes com íons da atmosfera densa na região cromosférica/fotosférica que ocorrem próximas do plano (xy) em $z=0$.
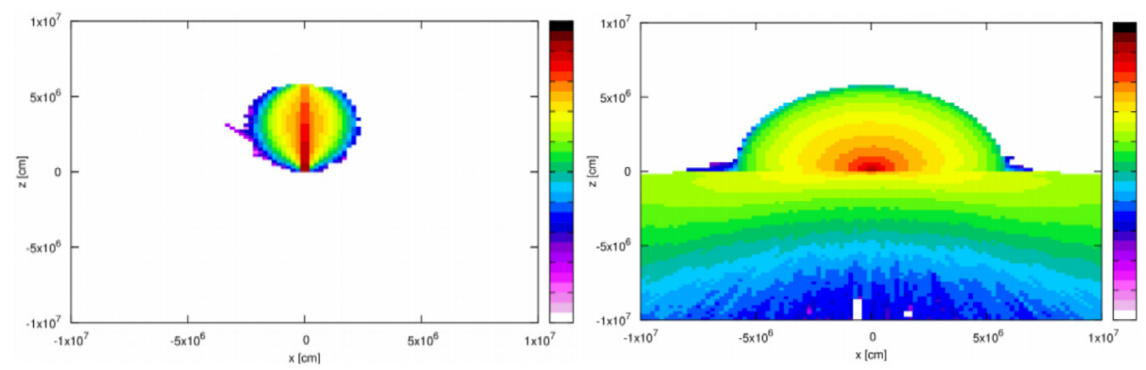

Figura 6 Distribuições espaciais de prótons primários, projetadas no plano (xz), obtidas para um feixe colimado (esquerda) e um feixe com distribuição semi-isotrópica (direita).

A Figura 7 mostra os espectros de fótons obtidos para um feixe de prótons primários colimado e um feixe com distribuição semi-isotrópica. Observamos que o fluxo de fótons obtido para o feixe com distribuição semi-isotrópica é mais intenso do que o obtido para o feixe colimado em todo o intervalo de energia. Também observamos que o espectro obtido para o feixe com distribuição semi-isotrópica se estende até energias de $\sim 0,7 \mathrm{GeV}$, enquanto o espectro obtido para o feixe colimado se estende até energias de $\sim 0,4 \mathrm{GeV}$. Esse resultado deve-se ao fato de que o feixe de prótons colimado produz uma fração maior de fótons a profundidades maiores, tal que o fluxo resultante é mais atenuado pelos processos de espalhamento Compton e produção de pares. 


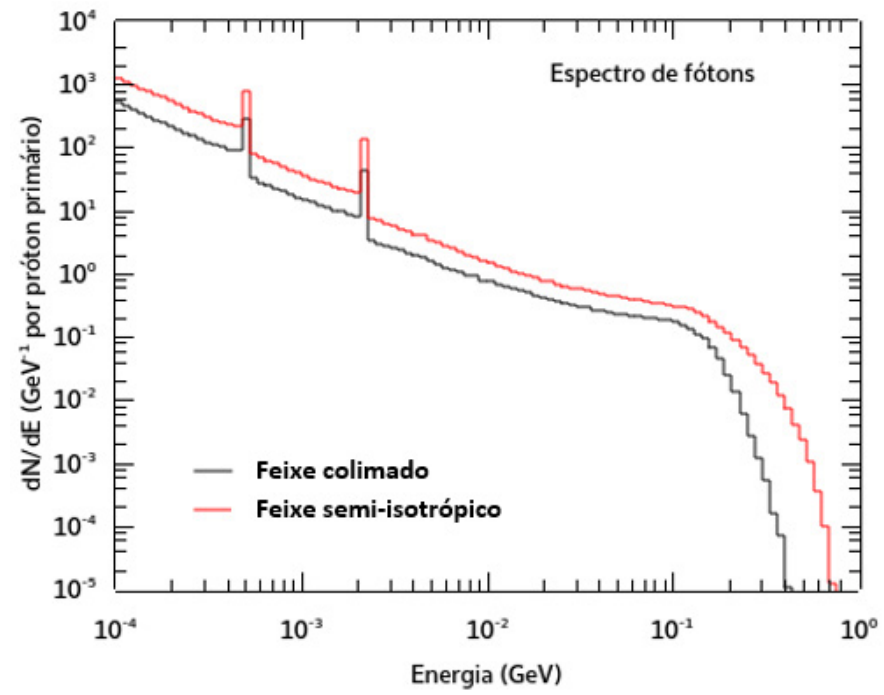

Figura 7 Espectros de fótons obtidos para um feixe de prótons primários colimado e um feixe de prótons primários com distribuição semi-isotrópica.

A Figura 8 mostra os espectros de fótons obtidos para um feixe de prótons primários monoenergéticos de $1 \mathrm{GeV}$ e um feixe de prótons primários com distribuição de energia do tipo lei de potência de índice espectral $\delta=2,2$ no intervalo de energia de 0,2 a $1 \mathrm{GeV}$. Em ambos os casos, consideramos o Modelo I para a atmosfera solar ambiente, um feixe com distribuição angular semi-isotrópica no hemisfério superior e um cenário de alvo espesso. Observamos que o fluxo de fótons obtido no caso do feixe de prótons primários monoenergéticos é mais intenso do que o obtido no caso do feixe com distribuição de energia do tipo lei de potência. Esse resultado é esperado, uma vez que a energia total injetada no alvo é maior no caso do feixe de prótons primários monoenergéticos de $1 \mathrm{GeV}$, de modo que um número maior de fótons é produzido. 


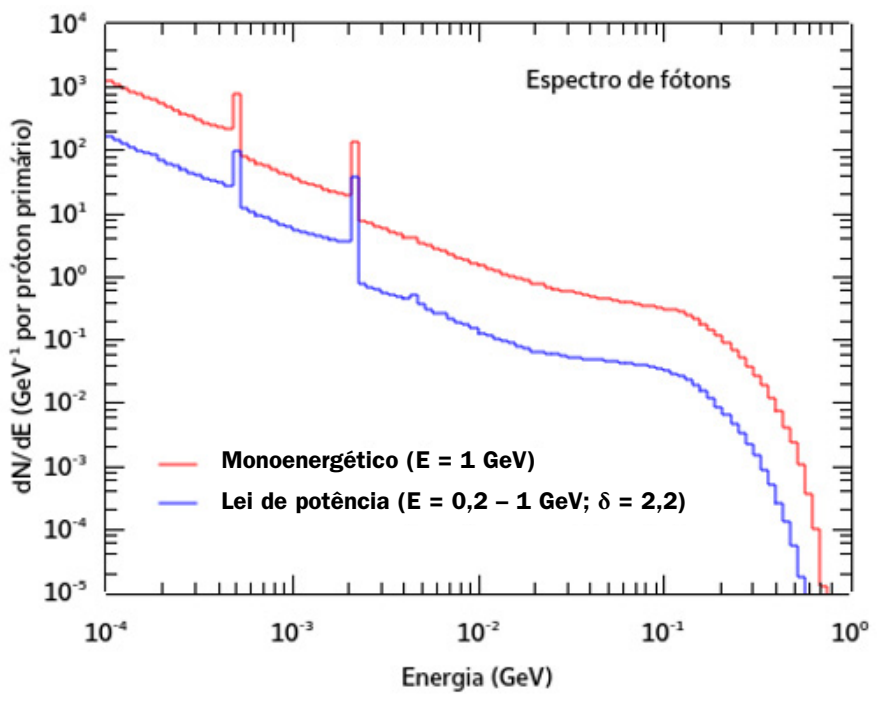

Figura 8 Espectros de fótons obtidos para um feixe de prótons primários monoenergéticos de $1 \mathrm{GeV}$ e um feixe de prótons primários com distribuição de energia do tipo lei de potência de índice espectral $\delta=2,2$ no intervalo de energia de 0,2 a $1 \mathrm{GeV}$.

Utilizando a funcionalidade do FLUKA que permite definir um limiar de energia abaixo do qual um dado processo é suprimido, analisamos o comportamento do espectro de fótons com relação aos processos de bremsstrablung de elétrons e pósitrons secundários, espalhamento Compton e decaimento de píons. Consideramos o Modelo I para a atmosfera solar ambiente, um feixe de prótons primários com distribuição angular semi-isotrópica e distribuição de energia do tipo lei de potência de índice espectral $\delta=2,2$ no intervalo de energia de 0,2 a $1 \mathrm{GeV}$ e um cenário de alvo espesso.

A Figura 9 mostra os espectros de fótons obtidos suprimindo-se o processo de bremsstrablung de elétrons e pósitrons secundários e o processo de espalhamento Compton, comparados com espectro total obtido na simulação incluindo todos os processos. Suprimindo-se o processo de bremsstrahlung de elétrons e pósitrons secundários, observamos uma redução do fluxo de fótons em energias abaixo de $\sim 40 \mathrm{MeV}$ (mais significativa no intervalo entre a linha de captura em 2,223 MeV e $~ 40 \mathrm{MeV}$ ). Notamos também que a contribuição devida à emissão de linhas de desexcitação nuclear no intervalo de energia de $\sim 4$ a $10 \mathrm{MeV}$ se torna mais visível. $\mathrm{Na}$ região de energias acima de $\sim 100 \mathrm{MeV}$, dominada pela emissão proveniente do decaimento de píons neutros, o espectro praticamente coincide com o obtido na simulação incluindo todos os processos. Conforme esperado, suprimindo-se o processo de espalhamento Compton observamos uma diminuição dos efeitos de transporte responsáveis 
pela atenuação do fluxo de fótons. Particularmente, notamos um aumento significativo da intensidade das linhas de aniquilação em $511 \mathrm{keV}$ e captura de nêutrons em 2,223 MeV, resultante da eliminação de suas respectivas caudas de comptonização em baixas energias, e aumento do fluxo de fótons no intervalo de energia entre a linha de captura e $\sim 40 \mathrm{MeV}$.

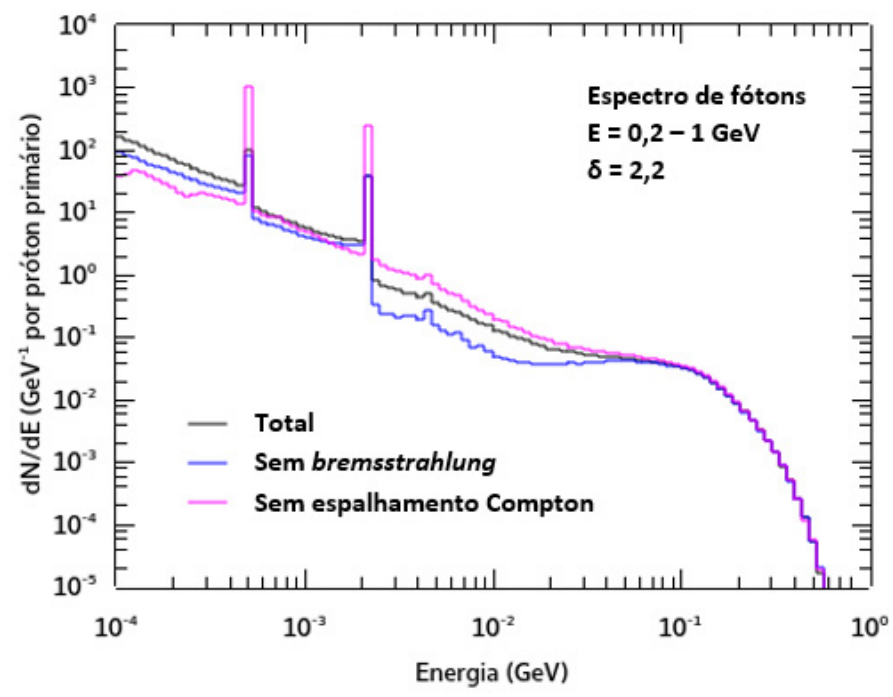

Figura 9 Espectros de fótons obtidos suprimindo-se o processo de bremsstrahlung de elétrons e pósitrons secundários e o processo de espalhamento Compton, comparados com o espectro total obtido na simulação incluindo todos os processos.

A Figura 10 mostra os espectros de fótons obtidos suprimindo-se o processo de decaimento de píons neutros e o processo de decaimento de píons carregados, comparados com o espectro total obtido na simulaçáo incluindo todos os processos. Conforme esperado, suprimindo-se os píons carregados, observamos uma redução do fluxo de fótons em energias abaixo de $\sim 100 \mathrm{MeV}$, uma vez que a maior parte dos elétrons e pósitrons secundários é eliminada. Também observamos uma significativa redução da intensidade da linha de aniquilação em $511 \mathrm{keV}$, uma vez que apenas os pósitrons secundários provenientes do decaimento Dalitz de píons neutros e da produção de pares contribuem para a formação da linha. Analogamente ao que ocorre quando o processo de bremsstrablung de elétrons e pósitrons secundários é suprimido, observamos que a contribuição das linhas de desexcitação nuclear no intervalo de energia de $\sim 4$ a $10 \mathrm{MeV}$ se torna mais visível. Suprimindo-se os píons neutros observamos, conforme esperado, uma significativa redução do fluxo de fótons em 
energias acima de $\sim 20 \mathrm{MeV}$ e ligeira redução do fluxo de fótons em energias abaixo de $\sim 20 \mathrm{MeV}$, uma vez que são eliminados os fótons produzidos pelo decaimento direto dos píons neutros.

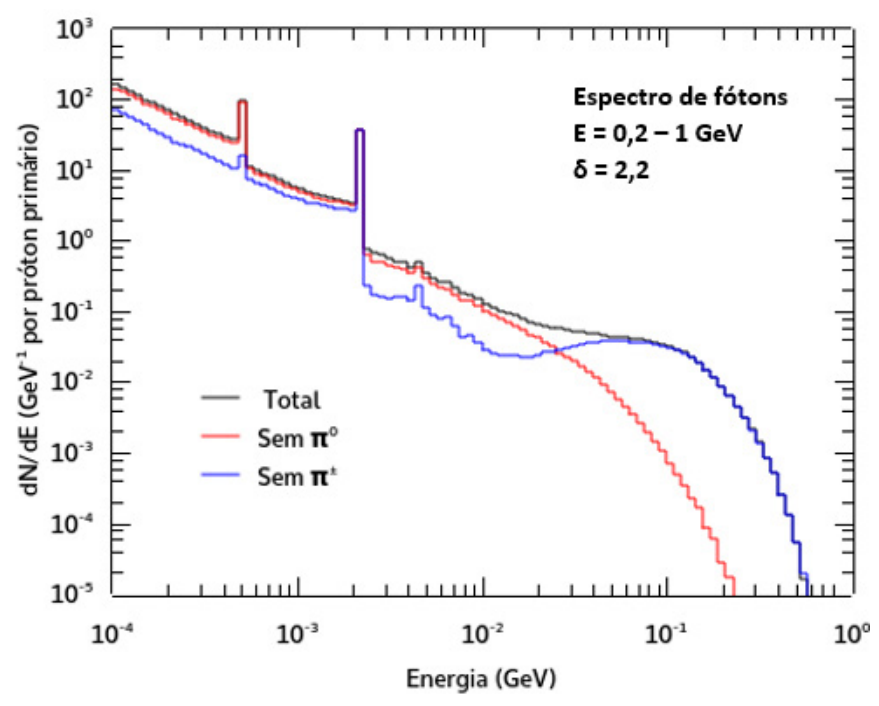

Figura 10 Espectros de fótons obtidos suprimindo-se o processo de decaimento de píons neutros e o processo de decaimento de píons carregados, comparados com o espectro total obtido na simulação incluindo todos os processos.

A Figura 11 mostra os espectros de fótons obtidos em simulaçóes realizadas com os Modelos I e II. Em ambos os casos consideramos um feixe de prótons primários com distribuição angular semi-isotrópica e distribuição de energia do tipo lei de potência de índice espectral $\delta=2,2$ no intervalo de energia de 0,2 a $1 \mathrm{GeV}$ e um cenário de alvo espesso. Conforme esperado, observamos que náo há diferenças significativas entre os espectros obtidos com os dois modelos, uma vez que, em um cenário de alvo espesso, os processos de emissão relevantes dependem essencialmente da profundidade de coluna correspondente ao range $R\left(E_{0}^{\max }\right)$ dos prótons primários de mais alta energia injetados, e não dos detalhes da estrutura vertical da atmosfera solar ambiente. 


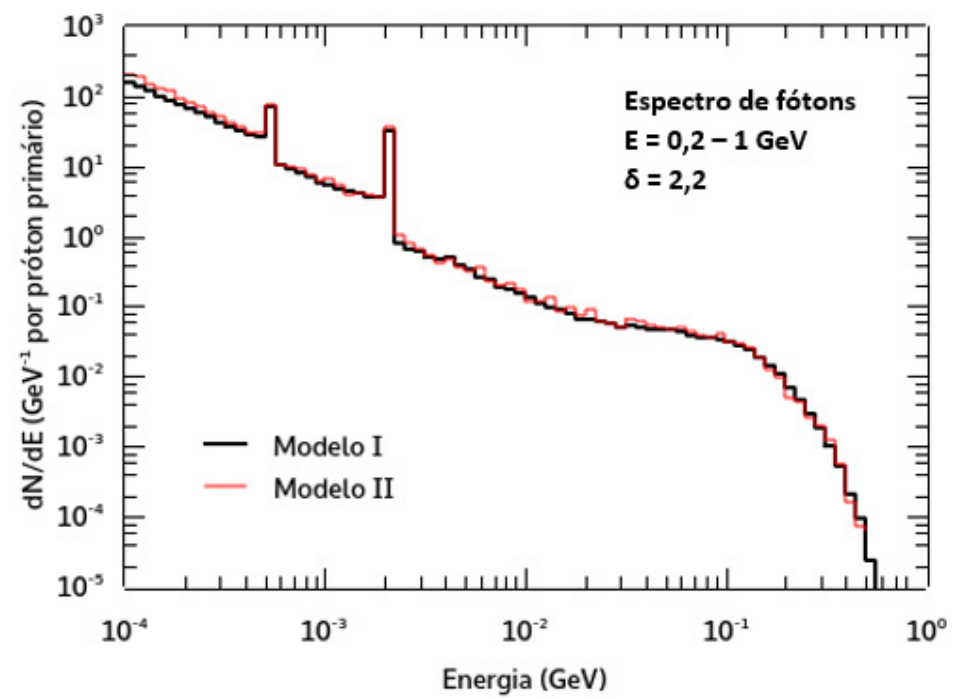

Figura 11 Espectros de fótons obtidos em simulações realizadas com os Modelos I e II.

\section{CONSIDERAÇÓES FINAIS}

Neste trabalho, apresentamos e discutimos os resultados de simulaçóes do espectro de emissão de raios gama em explosóes solares realizadas com o pacote Monte Carlo FLUKA. Considerando modelos simples para a atmosfera solar ambiente e feixes de prótons acelerados com diferentes distribuiçóes energéticas e angulares, obtivemos espectros de fótons exibindo as principais componentes dos espectros de emissão de raios gama observados em explosôes solares. Em trabalhos futuros, pretendemos desenvolver uma modelagem detalhada para descrever o transporte de íons primários acelerados em explosóes solares (prótons, partículas-alfa e núcleos mais pesados), bem como a produção e o transporte das partículas secundárias resultantes de processos nucleares (íons, nêutrons, píons, fótons, elétrons e pósitrons), visando à elaboração de ferramentas teóricas para a análise de dados de observaçóes de explosôes solares na faixa de raios gama. 


\title{
SIMULATIONS OF NUCLEAR PROCESSES IN SOLAR FLARES USING THE MONTE CARLO PACKAGE FLUKA
}

\begin{abstract}
The emission of gamma-rays in solar flares is produced by interactions of electrons and ions accelerated to high energies with nuclei of the ambient solar atmosphere. The analysis of the gamma-ray emission spectrum observed in solar flares provides important diagnostics both on the mechanisms of particle acceleration and the structure and evolution of the ambient plasma. In this work we present and discuss the results of simulations of high energy nuclear processes in solar flares using the Monte Carlo package FLUKA. Considering simple models for the ambient solar atmosphere and beams of accelerated protons with different energy and angular distributions we obtained gamma-ray emission spectra similar to those observed of the solar flares.
\end{abstract}

Keywords: Solar Flare. Nuclear Processes. FLUKA.

\section{REFERÊNCIAS}

ANDERSEN, V. et al. The FLUKA code for space applications: recent developments. Advances in Space Research, v. 34, n. 6, p. 1302-1310, 2004. Disponível em: http://www0.mi.infn. it/ -garzelli/pub/AdvSpaRes34-1302.pdf. Acesso em: 13 ago. 2021.

ASCHWANDEN, M. J. Physics of the solar corona: an introduction. [S.l.]: Praxis Publishing Ltd., 2005.

ASPLUND, M. et al. The chemical composition of the Sun. Annual Review of Astronomy and Astrophysics, v. 47, n. 1, p. 481-522, Sept. 2009. Disponível em: https://arxiv.org/pdf/0909.0948. pdf. Acesso em: 13 ago. 2021.

BASTIAN, T.; BENZ, A.; GARY, D. Radio emission from solar flares. Annual Review of Astronomy and Astrophysics, v. 36, n. 1, p. 131-188, 1998.

BATTISTONI, G. et al. Recent developments in the FLUKA nuclear reaction models. In: INTERNATIONAL CONFERENCE ON NUCLEAR REACTION MECHANISMS, 11. 2006, Varenna. Proceedings [...]. Varenna: University of Milano, 2006. p. 483-495. Disponível em: http://www0.mi.infn.it/ -gadioli/Varenna2006/Proceedings/Ferrari_A.pdf. Acesso em: 13 ago. 2021. 
BATTISTONI, G. et al. Overview of the FLUKA code. Annals of Nuclear Energy, v. 82, p. 10-18, Aug. 2015. Disponível em: https://www.sciencedirect.com/science/article/pii/ S0306454914005878. Acesso em: 9 ago. 2021.

BERGER, M. J. et al. ESTAR, PSTAR and ASTAR: computer programs for calculating stopping-power and range tables for electrons, protons and helium ions. National Institute of Standards and Technology, 2005. Disponível em: http://www.nist.gov/pml/data/star/. Acesso em: 2 ago. 2021.

BETHE, H. A. Moliére's theory of multiple scattering. Physical Review Journals Archive, v. 89, n. 6, p. 1256-1266, Mar. 1953. Disponível em: https://journals.aps.org/pr/abstract/10.1103/ PhysRev.89.1256. Acesso em: 9 ago. 2021.

BETHE, H. A.; HEITLER, W. On the stopping of fast particles and on the creation of positive electrons. Proceedings of the Royal Academy, v. 146, n. 856, p. 83-112, Aug. 1934. Disponível em: https://royalsocietypublishing.org/doi/10.1098/rspa.1934.0140. Acesso em: 9 ago. 2021.

CAPELLA, A. et al. Dual parton model. Physics Reports, v. 236, n. 4-5, p. 225-329, Jan. 1994. Disponível em: https://www.sciencedirect.com/science/article/pii/0370157394900647?via\%3 Dihub. Acesso em: 9 ago. 2021.

CERUTTI, F. et al. Low energy nucleus-nucleus reactions: the BME approach and its interface with FLUKA. In: INTERNATIONAL CONFERENCE ON NUCLEAR REACTION MECHANISMS, 11., 2006, Varenna. Proceedings [...]. Varenna: University of Milano, 2006. p. 1-8. Disponível em: http://www0.mi.infn.it/-gadioli/Varenna2006/Proceedings/Cerutti_F. pdf. Acesso em: 13 ago. 2021.

DULK, G. A.; MARSH, K. A. Simplified expressions for the gyrosynchrotron radiation from mildly relativistic, nonthermal and thermal electrons. The Astrophysical Journal, v. 259, p. $350-$ 358, Aug. 1982. Disponível em: http://articles.adsabs.harvard.edu/pdf/1982ApJ...259..350D. Acesso em: 13 ago. 2021.

FASSÒ, A. et al. FLUKA: performances and applications in the intermediate energy range. In: SPECIALISTS' MEETING ON SHIELDING ASPECTS OF ACCELERATORS, TARGETS AND IRRADIATION FACILITIES, 1., 1994, Arlington. Proceedings [...]. Arlington: OECD Documents, 1994. p. 287-304. Disponível em: https://cds.cern.ch/record/2724814/ files/fulltext.pdf. Acesso em: 13 ago. 2021.

FERRARI, A.; SALA, P. R. A new model for hadronic interactions at intermediate energies for the FLUKA code. In: INTERNATIONAL CONFERENCE ON MONTE CARLO SIMULATION IN HIGH-ENERGY AND NUCLEAR PHYSICS, 1993, Milan. Proceedings [...]. Milan: 1994. p. 277-288. Disponível em: http://www.fluka.org/content/publications/1993_ mc_preq.pdf. Acesso em: 13 ago. 2021.

FERRARI, A.; SALA, P. R. The physics of high energy reactions. In: WORKSHOP ON NUCLEAR REACTION DATA AND NUCLEAR REACTORS PHYSICS, DESIGN AND SAFETY, Trieste, 1996. Proceedings [...]. Trieste: World Scientific, 1998. p. 424-532. Disponível em: https://inis.iaea.org/search/searchsinglerecord.aspx?recordsFor=SingleRecord\& $\mathrm{RN}=29067414$. Acesso em: 13 ago. 2021.

FERRARI, A.; SALA, P. R.; FASSÒ, A.; RANFT, J. FLUKA: A Multi-Particle Transport Code. Stanford: Stanford University, 2005. Disponível em: https://inspirehep.net/files/ e8cb3847561a920327bd8b03bc518617. Acesso em: 13 ago. 2021. 
FERRARI, A.; SALA, P. R.; GUARALDI, R.; PADOANI, F. An improved multiple scattering model for charged particle transport. Elsevier Science Publishers B. V., v. 71, n. 4, p. 412-426, Sept. 1992. Disponível em: https://www.sciencedirect.com/science/article/pii/0168583X9295359Y. Acesso em: 13 ago. 2021.

GETACHEW, A. Stopping power and range of protons of various Energies in different materials. 2007. 50 f. Dissertação (Mestrado em Física) - Faculty of Science, Addis Ababa University, 2007. Disponível em: http://etd.aau.edu.et/bitstream/handle/123456789/404/Abebe\%20Getachew.pdf?sequence=1\&isAllowed=y. Acesso em: 13 ago. 2021.

HUA, X. M. et al. Angular and energy-dependent neutron emission from solar flare magnetic loops. The Astrophysical Journal Supplement Series, v. 140, n. 2, p. 563-579, Jun. 2002. Disponível em: https://iopscience.iop.org/article/10.1086/339372/pdf. Acesso em: 13 ago. 2021.

KOZLOVSKY, B.; LINGENFELTER, R. E.; RAMATY, R. Positrons from accelerated particle interactions. The Astrophysical Journal, v. 316, p. 801-818, May 1987. Disponível em: http:// articles.adsabs.harvard.edu/pdf/1987ApJ...316..801K. Acesso em: 13 ago. 2021.

KOTOKU, J. et al. Effects of Compton scattering on the gamma ray spectra of solar flares. Astronomical Society of Japan, v. 59, n. 6, p. 1161-1174, Dec. 2007. Disponível em: https:// academic.oup.com/pasj/article/59/6/1161/1514621. Acesso em: 13 ago. 2021.

SHEN, B. S. P. (ed.). High-energy nuclear reactions in astrophysics. New York: W. A. Benjamin, 1967.

MACKINNON, A.; SZPIGEL, S.; DE CASTRO, C. G. G.; TUNEU, J. FLUKA simulations of pion decay gamma-radiation from energetic flare ions. Solar Physics, v. 295, n. 12, Dec. 2020. Disponível em: https://arxiv.org/pdf/2009.00414.pdf. Acesso em: 13 ago. 2021.

MORRISON, P. On gamma-ray astronomy. Il Nuovo Cimento, v. 7, n. 6, p. 858-865, Mar. 1958. Disponível em: https://link.springer.com/article/10.1007\%2FBF02745590. Acesso em: 13 ago. 2021.

MURPHY, R.; DERMER, C. D.; RAMATY, R. High-energy processes in solar flares. The Astrophysical Journal Supplement Series, v. 63, p. 721-748, Mar. 1987. Disponível em: http:// articles.adsabs.harvard.edu/pdf/1987ApJS...63..721M. Acesso em: 16 ago. 2021.

MURPHY, R. J. et al. The physics of positron annihilation in the solar atmosphere. The Astrophysical Journal Supplement Series, v. 161, n. 2, p. 495-519, Dec. 2005. Disponível em: https:// iopscience.iop.org/article/10.1086/452634/pdf. Acesso em: 16 ago. 2021.

MURPHY, R. J. et al. Using gamma-ray and neutron emission to determine solar flare accelerated particle spectra and composition and the conditions within the flare magnetic loop. The Astrophysical Journal Supplement Series, v. 168, p. 167-194, Jan. 2007. Disponível em: https:// iopscience.iop.org/article/10.1086/509637/pdf. Acesso em: 16 ago. 2021.

OLIVE, K. A. Review of particle physics. Chinese Physics, v. 38, n. 9, 2014. Disponível em: http://stacks.iop.org/1674-1137/38/i=9/a=090001. Acesso em: 2 ago. 2021.

RAMATY, R. Astrophysical gamma ray lines from accelerated particle interactions. In: SIGNORE, M.; SALATI, P.; VEDRENNE, G. (ed.). Gamma Ray Sky with Compton GRO and SIGMA. [S. l.]: Springer, 1995. p. 279-301.

RAMATY, R.; KOZLOVSKY, B.; LINGENFELTER, R. E. Nuclear gamma-rays from energetic particle interactions. The Astrophysical Journal Supplement Series, v. 40, p. 487-526, Jul. 
1979. Disponível em: http://articles.adsabs.harvard.edu/pdf/1979ApJS...40..487R. Acesso em: 16 ago. 2021.

RAMATY, R.; MURPHY, R. J. Nuclear processes and accelerated particles in solar flares. Space Science Review, v. 45, n. 3-4, p. 213-268, Jan. 1987. Disponível em: https://link.springer. com/article/10.1007/BF00171995. Acesso em: 16 ago. 2021.

ROESLER, S.; ENGEL, R.; RANFT, J. The Monte Carlo event generator DPMJET-III. In: INTERNATIONAL CONFERENCE ON ADVANCED MONTE CARLO FOR RADIATION PHYSICS, PARTICLE TRANSPORT SIMULATION AND APPLICATIONS, 2000, Lisbon. Proceedings [...]. Lisbon: Springer, 2000. p. 1033-1038. Disponível em: https:// arxiv.org/pdf/hep-ph/0012252.pdf. Acesso em: 16 ago. 2021.

SORGE, H.; STÖCKER, H.; GREINER, W. Poincaré invariant Hamiltonian dynamics: modelling multi-hadronic interactions in a phase space approach. Annals of Physics 192, n. 2, p. 266-306, Jun. 1989. Disponível em: https://www.sciencedirect.com/science/article/abs/pii/ 000349168990136X?via\%3Dihub. Acesso em: 16 ago. 2021.

TANDBERG-HANSSEN, E.; EMSLIE, G. The physics of solar flares. [S.l.]: Cambridge University Press, 2009.

VILMER, N.; MACKINNON, A. L.; HURFORD, G. J. Properties of energetic ions in the solar atmosphere from $\gamma$-ray and neutron observations. Space Science Reviews, v. 159, n. 1, p. 167-224, Oct. 2011. Disponível em: https://arxiv.org/pdf/1110.2432.pdf. Acesso em: 16 ago. 2021.

\section{AGRADECIMENTOS}

Este trabalho foi realizado com o apoio financeiro da Fundação de Amparo à Pesquisa de São Paulo (Fapesp) mediante Auxílio à Pesquisa - Regular (processo 2017/13282-5). Um dos autores, D. S. Tusnski, recebeu apoio do Instituto Presbiteriano Mackenzie por meio de bolsa de doutorado na modalidade de isenção de taxas escolares.

\section{Contato}

Sergio Szpigel

szpigel@mackenzie.br

\section{Tramitação}

Recebido em dezembro de 2020.

Aprovado em fevereiro de 2021. 